\title{
Determination of Weber-Ampere Characteristics of Electric Devices Using Solution of Inverse Problem of Harmonic Balance
}

\author{
Anton Mikhailovich Lankin ${ }^{1}$, Mikhail Vladimirovich Lankin ${ }^{1}$, Nikolay Ivanovich Gorbatenk ${ }^{1} \&$ \\ Danil VadimovichShaykhutdinov ${ }^{1}$ \\ ${ }^{1}$ Platov South-Russian State Polytechnic University (NPI), Russia \\ Correspondence: Lankin Anton Mikhailovich, Platov South-Russian State Polytechnic University (NPI), Russia.
}

Received: January 15, $2015 \quad$ Accepted: February 3, $2015 \quad$ Online Published: July 30, 2015

doi:10.5539/mas.v9n8p247 URL: http://dx.doi.org/10.5539/mas.v9n8p247

\begin{abstract}
This work is devoted to development of measurement of weber--ampere characteristic of electric devices consisting of a magnetic core and a coil. In this method the coil of electric device is energized with sinusoidal voltage of known amplitude and frequency, and harmonic amplitudes of passing current are measured. On the basis of these data the inverse problem of harmonic balance is solved and the approximation coefficients of the equation describing the required weber--ampere characteristic are determined. The influence of the degree of approximating expression on measurement error is studied. The obtained results have demonstrated possibility of application of the proposed method to measurements of weber--ampere characteristics of electric devices. The measurement error of weber--ampere characteristic does not exceed $3 \%$.
\end{abstract}

Keywords: weber - ampere characteristic, electric device, measurement methods, harmonic balance, weber ampere characteristic of operating cycle

\section{Introduction}

The miniaturization trend and attempts to decrease materials consumption of up-to-date electric devices present increased requirements to quality of their individual elements, which can be estimated on the basis of their mechanical, electrical and magnetic properties. Each of the aforementioned groups of indices require for application of dedicated methods and tools of testing of electric devices. This situation promotes searching for integral indices of operation of electric devices, which can provide conclusions about quality of separate parts and operational characteristics of overall product.

All electric devices (electric magnets, electromagnetic relays, motors) include moving and static parts of magnetic core and at least one operating coil (Korotyeyev, Zhuikov, Kasperek, 2010). Within operation of an electric device current flows through operating coil, working magnetic flow is generated in the static part of magnetic core, put in motion its moving part. The value of magnetic flow is determined by design and mutual position of part of magnetic core and operating coil, as well as the number of turns in operating coil and flowing current. Within operating cycle of electric device the moving part of magnetic core travels with regard to the static part, which also varies magnetic flow. All this suggests that the integral characteristic, providing information not only about operating parameters of electric device but also about quality of its individual parts, is weber - ampere characteristic of operating cycle. Weber--ampere characteristic of operating cycle is the magnetic flow passing through operating coil of electric device as a function of current flowing via the coil. Herewith, the moving part pf magnetic core of electric device executes typical operating movement. In order to obtain weber--ampere characteristic of an electric device it is necessary to apply external varying magnetic field to magnetic core and to measure magnetic flow in its cross section by means of special sensor (Singh, 2003; ASTM International, 1970; Kiefer, 1969). In order to obtain weber--ampere characteristic of operating cycle electric device in assembly is tested, thus, it is impossible to use sensors of magnetic field.

Now let us consider measurement tools of weber--ampere characteristic, where the source of internal magnetic field is the coil of electric device, and the flow is determined indirectly.

For instance, substitution method, where the circuit of sinusoidal voltage source is alternatively connected either with the coil applied on magnetic core of tested electric device, or known variable inductance $L_{0}$ and non-reactive resistance $r$. In addition, ammeter and variable inductance $L$ and capacitor $C$ are connected in series 
with the circuit of sinusoidal voltage source. Initially, with known value of output voltage $U$ of the sinusoidal voltage source it is connected with the coil of tested electric device and by means of variable inductance $L$ and capacitor $C$, the circuit is adjusted to resonance which is detected by maximum value of current $I$ in magnetizing circuit. Then the circuit, instead of winding of tested device, is connected with known variable inductance $L_{0}$ and non-reactive resistance $r$ and by means thereof the circuit is again adjusted to resonance. Herewith, by variation of non-reactive resistance $r$, the same value of current $I$ in the circuit is obtained (the values $L$ and $C$ are not modified). Within resonance the inductance of winding $L_{\mathrm{x}}$, applied to the tested device, equals to know variable inductance $L_{0}$. When it is known, inductance $B$ or flow of magnetic field $\Phi$ in the tested device are calculated as follows:

$$
\Phi=\frac{L_{0} \cdot I \cdot \sqrt{2}}{4 \pi w}
$$

where $I$ is the current measured by ammeter, $w$ is the number of coils of the tested device.

Another values of output voltage of the sinusoidal voltage source $U$ are set, and measuring the values of current $I$ in magnetizing circuit the magnetic flow $\Phi$ is calculated, thus obtaining the required number of points of weber--ampere characteristic.

A drawback of this method is that acceptable accuracy is provided only with sinusoidal variation of magnetizing current, in the region of small magnetic fields, and within saturation of the tested device, when current becomes non-sinusoidal, the calculation by Eq. (1) results in high error.

Another method of measurement of weber--ampere characteristic of electric device involves connection of the coil of electric device to the sinusoidal voltage source, then the voltage and current are measured on the coil of electric device, and the coordinates of weber--ampere characteristic are calculated as the integral of difference between the voltage supplied to the coil of electric device and voltage drop at its active resistance:

$$
\Phi=\frac{1}{k} \int(U-I R) d t
$$

where $U$ is the voltage at the output of sinusoidal voltage source, $I$ is the current flowing through the coil of electric device; $R$ is the active component of circuit resistance of coil of electric device; $k$ is the coefficient determined by the number of windings of coil of electric device, length of median line and surface area of transversal cross section of magnetic core of electric device. The active component of resistance $R$ of coil of electric device, including the active portion of resistance of the coil of electric device, resistance of current instrument shunt and output resistance of sinusoidal voltage source are preliminary detected and then applied as a constant within calculation of magnetic flow, however, during the measurements as a consequence of current passing through the coil of electric device it is heated and its active resistance $R$ increases. Hence, Eq. (2) works incorrectly, which adds significant and accumulating within integration error to the measurements.

A drawback of the method is that it is based on integration, and the coil active resistance varies within its heating. The necessity to eliminate these factors initiated several works, which more or less successfully solve this problem Roller, 2012; Sakhavova, Shirokov, and Yanvarev, 2013).

Application of the existing methods for measurement of weber--ampere characteristic of operating cycle of electric device has some drawbacks, thus, it is required to develop a method for measurement of weber--ampere characteristic of operating cycle of electric device with sufficient accuracy and without the aforementioned drawbacks.

\section{Methodology}

\subsection{The Essence of Harmonic Balance}

The problems of harmonic balance are applied for investigation into various non-linear circuits (Bessonov, 1978; Song Xing, Suting Chen, Zhanming Wei, Jingming Xia, 2013). Direct problem of harmonic balance consists of determination of shape of current passing through non-linear element at its known volt--ampere characteristic, amplitude and shape of feeding voltage. The essence of the method is based on decomposition of periodical functions of voltage applied to non-linear element and current flowing through it into Fourier series. In general case the unknown variables in non-linear electric circuit are non-sinusoidal and contain infinite spectrum of harmonics. The expected solution can be presented as the sum of main and several higher harmonics. Substituting this sum into non-linear differential equation compiled for the required variable and setting equal the coefficients preceding harmonics in the obtained expression (sinusoidal and non-sinusoidal functions) of the equal frequencies in its left- and right-hand sides, we obtain a set of $n$ algebraic equations, where $n$ is the number 
of considered harmonics. Solving the set of equations we obtain the unknown variables. The developed method is based on inverse problem of harmonic balance, which consists of determination of unknown volt--ampere characteristic and non-linear element qith known shape of current flowing through it, shape and amplitude of feeding voltage.

\subsection{Computation Algorithm of Direct and Inverse Problems of Harmonic Balance}

Let us consider solution of direct problem of harmonic balance for the case of non-linear circuit consisting orf sinusoidal voltage source and non-linear resistance (Figure 1).

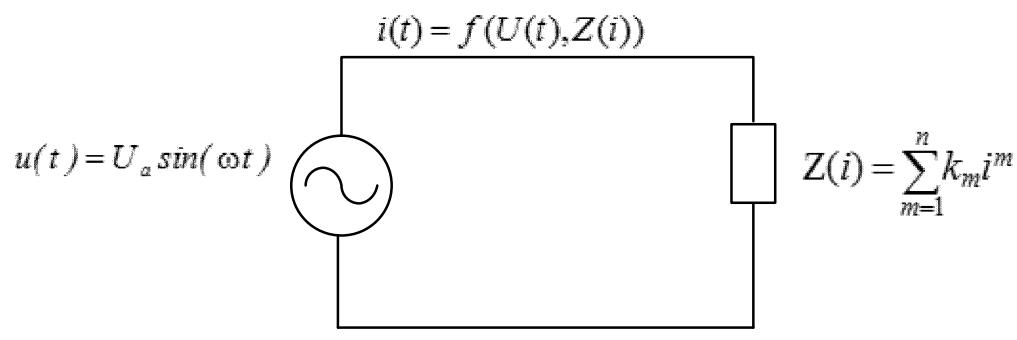

Figure 1. Schematic presentation of problems of harmonic balance

Solution of direct problem of harmonic balance includes the following steps:

1. Equations of circuit (Figure 1) are written for instant values.

$$
u(t)=i(t) \cdot Z(i)
$$

where $u(t)=U_{a} \sin (\omega t), \quad i(t)$ are the instant values of voltage and current; $Z(i)$ is the total resistance, $U_{a}$ is the voltage amplitude, $\omega$ is the angular frequency.

2. Expression of analytic approximation of non-linear dependence of total resistance of non-linear element on current is written:

$$
Z(i)=\sum_{m=0}^{n} k_{2 m+1} i^{2 m+1},
$$

where $k_{2 m+1}$ are the coefficients of approximating expression.

3. On the basis of preliminary analysis of circuit and non-linear characteristic (4) the equation of required value in the form of finite Fourier series is set (Tolstov, 2012) with unknown at this stage amplitudes of current harmonics $I_{2 m+1}$ :

$$
i(t)=\sum_{m=0}^{n} I_{2 m+1} \sin ((2 m+1) \omega t),
$$

where $I_{2 m+1}$ are the amplitudes of current harmonics. Equation (5) contains no even harmonics of current and components of cosine portion of decomposition into Fourier series due to symmetry of voltage--ampere characteristic with regard to origin of coordinates.

4. Equations (4) and (5) are substituted into equation of circuit (3), trigonometric transformations are carried out (Lopez, 1994) and the terms in the obtained equations are grouped according to numbers of harmonics:

$$
U_{a} \sin (\omega t)=\sum_{m=0}^{n} k_{2 m+1}\left(I_{2 m+1} \sin ((2 m+1) \omega t)\right)^{2 m+1} \sum_{m=0}^{n} I_{2 m+1} \sin ((2 m+1) \omega t) .
$$

A set of non-linear equations is compiled by substitution of various arguments $(\omega t)$ into Eq. (6). The obtained set of equations is solved with regard to unknown amplitudes of current harmonics $I_{2 m+1}$.

In certain cases it is necessary to determine not the current shape but analytic approximation of non-linear dependence of total resistance of non-linear element on current, then we apply the solution of inverse problem of harmonic balance. It differs in that the coefficients $k_{2 m+1}$ in Eq. (4) are unknown, and the amplitudes of current harmonics $I_{2 m+1}$ are known. therefore, the set compiled of Eq. (6) is solved with regard to $k_{2 m+1}$. 


\subsection{Application of Inverse Problem of Harmonic Balance for Determination of Weber-Ampere Characteristic of Electric Device}

Solution of direct problem of harmonic balance makes it possible to determine the shape of current $i(t)$, flowing through the coil of electric device, given in the form of non-linear inductance (Enns, McGuire, 2000; Enns, 2010) by means of decomposition into Fourier series:

$$
i(t)=\sum_{m=0}^{n} I_{(2 m+1)} \sin ((2 m+1) \omega t),
$$

where $I_{(2 m+1)}$ is the amplitude of the $(2 m+1)$-th current harmonic. Herewith, the shape and amplitude $U_{a}$ of voltage applied to non-linear inductance are known:

$$
u(t)=U_{\mathrm{a}} \sin (\omega t),
$$

as well as weber--ampere characteristic of non-linear inductance set by approximating equation:

$$
\Phi(i)=\sum_{m=0}^{n} k_{(2 m+1)} i^{2 \mathrm{~m}+1},
$$

where $\Phi$ is the value of magnetic flow through non-linear inductance, $k_{(2 m+1)}$ are the coefficients of approximating expression of weber--ampere characteristic, $m=\overline{(0, n)},(n+1)$ is the number of components in the approximating expression, $i$ is the current intensity through non-linear inductance.

The inverse problem of harmonic balance for determination of weber--ampere characteristic of electric device is formed as follows. There exists non-linear inductance with unknown weber--ampere characteristic, the regularities of variation of voltage (8) applied to non-linear inductance and flowing through it current (7) are known. It is required to determine the coefficients $k_{(2 m+1)}$ of approximating expression of weber--ampere characteristic (9).

Now let us write the equation of circuit with non-linear inductance involving active resistance $R$ :

$$
u(t)=R i+\frac{d \Phi}{d t} .
$$

Let us rewrite it with consideration for known regularities of variation of current (7) and voltage (8):

$$
U_{a} \sin \omega t=R \sum_{m=0}^{n} I_{(2 m+1)} \sin ((2 m+1) \omega t)+\frac{d \sum_{m=0}^{n} k_{(2 m+1)}\left(I_{(2 m+1)} \sin ((2 m+1) t \omega)\right)^{2 m+1}}{d t}
$$

On the basis of known degree $(2 n+1)$ of approximating expression of weber--ampere characteristic we set the $(n+1)$ value of sine function argument in Eq. (10). The argument value is taken from the interval ]0; $\pi / 2[$.

Therefore, we obtain a set of $(n+1)$ linear equations. The obtained set of equations contains known voltage amplitude $U_{a}$, amplitudes of current harmonics $I_{(2 m+1)}$, values of active resistance $R$ and circular frequency of flowing current $\omega$, since these parameters of circuit with non-linear inductance can be measured within testing of electric devices. Solving this set of equation we obtain coefficients $k_{(2 m+1)}$ of Eq. (9) approximating weber--ampere characteristic.

\section{Results Discussion}

\subsection{Simulation Experiment}

In order to implement the model it was necessary to perform certain requirements to the applied package of general-circuit simulation: possibility to set the required weber--ampere characteristic by points, plotting of spectra of periodic functions (current spectra), possibility of step-by-step reproduction of circuit, plotting of several function in one plot. All aforementioned requirements are satisfied by the MicroCap simulator (Vester, 2009; Amelina, Amelin, 2007).

A peculiar feature of numerous electric devices is the existence of non-magnetic gap in their magnetic cores. The model includes weber--ampere characteristic of magnetic core of material $3100 \mathrm{~V}$, for which two variants of non-magnetic gap are preset: $0.4 \mathrm{~mm}$ and $0.9 \mathrm{~mm}$, which leads to various slopes of weber--ampere characteristic.

The model of electric device contains connected in series non-linear inductance with 95 windings and active resistance $R$ of $0.15 \Omega$ and $0.1 \Omega$ for the aforementioned non-magnetic gaps, respectively. The electric device is connected to sinusoidal voltage source with frequency of $50 \mathrm{~Hz}$ and amplitude $U_{a}$ of $1.65 \mathrm{~V}$ and $1.7 \mathrm{~V}$ for the aforementioned non-magnetic gaps, respectively. 
Figure 2 illustrates the currents of в coils of electric device with selected non-magnetic gaps.

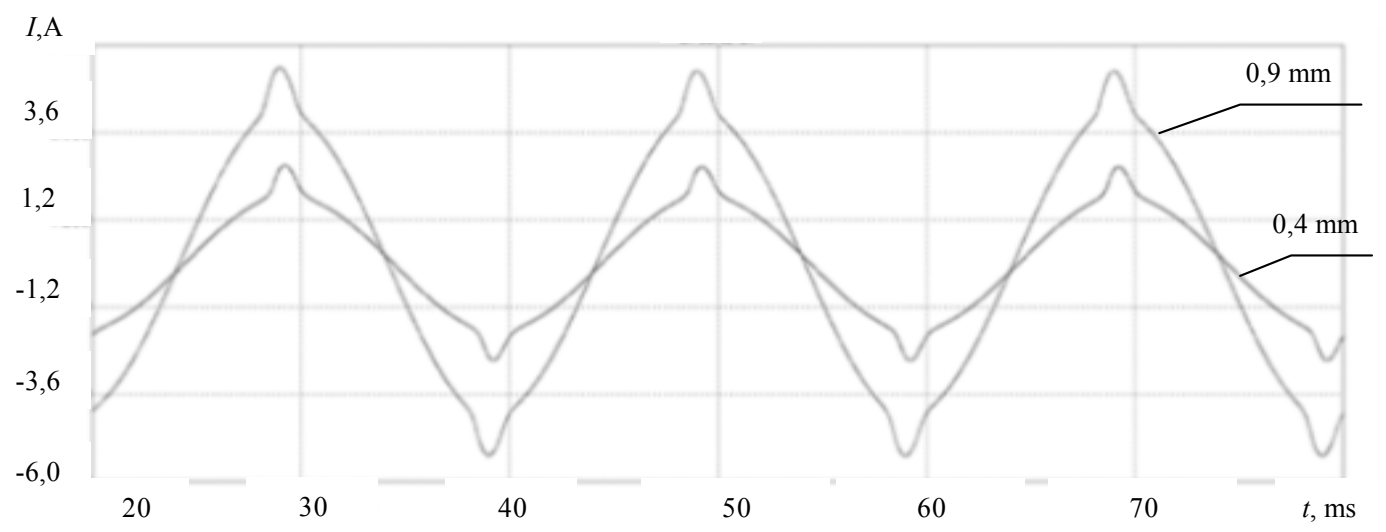

Figure 2. Currents in the coils of electric device

In Figure 2 the currents flowing through the coil of electric device are of non-sinusoidal shape, which can be attribute to the existence of non-linear element in the circuit.

Figures 3 and 4 illustrates the current spectra for selected non-magnetic gaps.

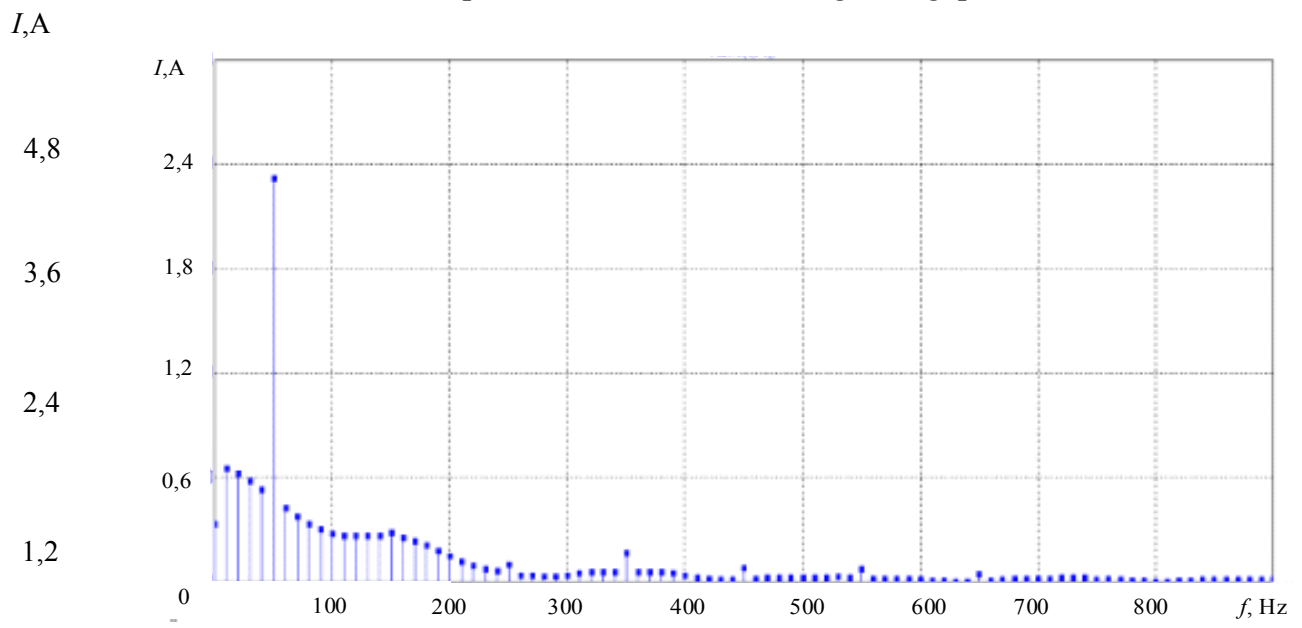

0

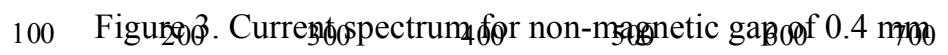

800

$f, \mathrm{~Hz}$

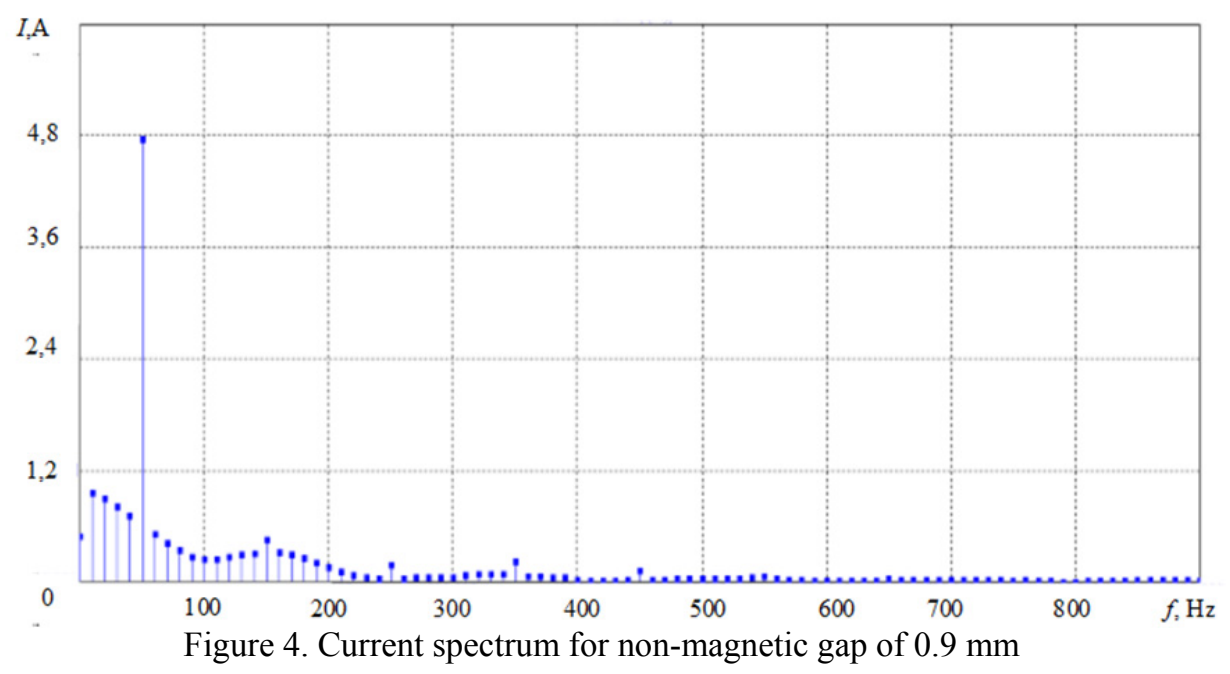


In Figs. 3 and 4 it can be seen that in addition to the first harmonic it is possible to determine higher harmonics from the first to the thirteenth ones. Herewith, the harmonics of current flowing through electric device with the gap of $0.9 \mathrm{~mm}$ have higher amplitude than the those through electric device with the gap of $0.4 \mathrm{~mm}$.

Determination of calculated weber--ampere characteristics is carried out by means of the proposed method on the basis of solution of inverse problem of harmonic balance, the results are illustrate din Figure 5, and the number of considered harmonics is given in parenthesis.

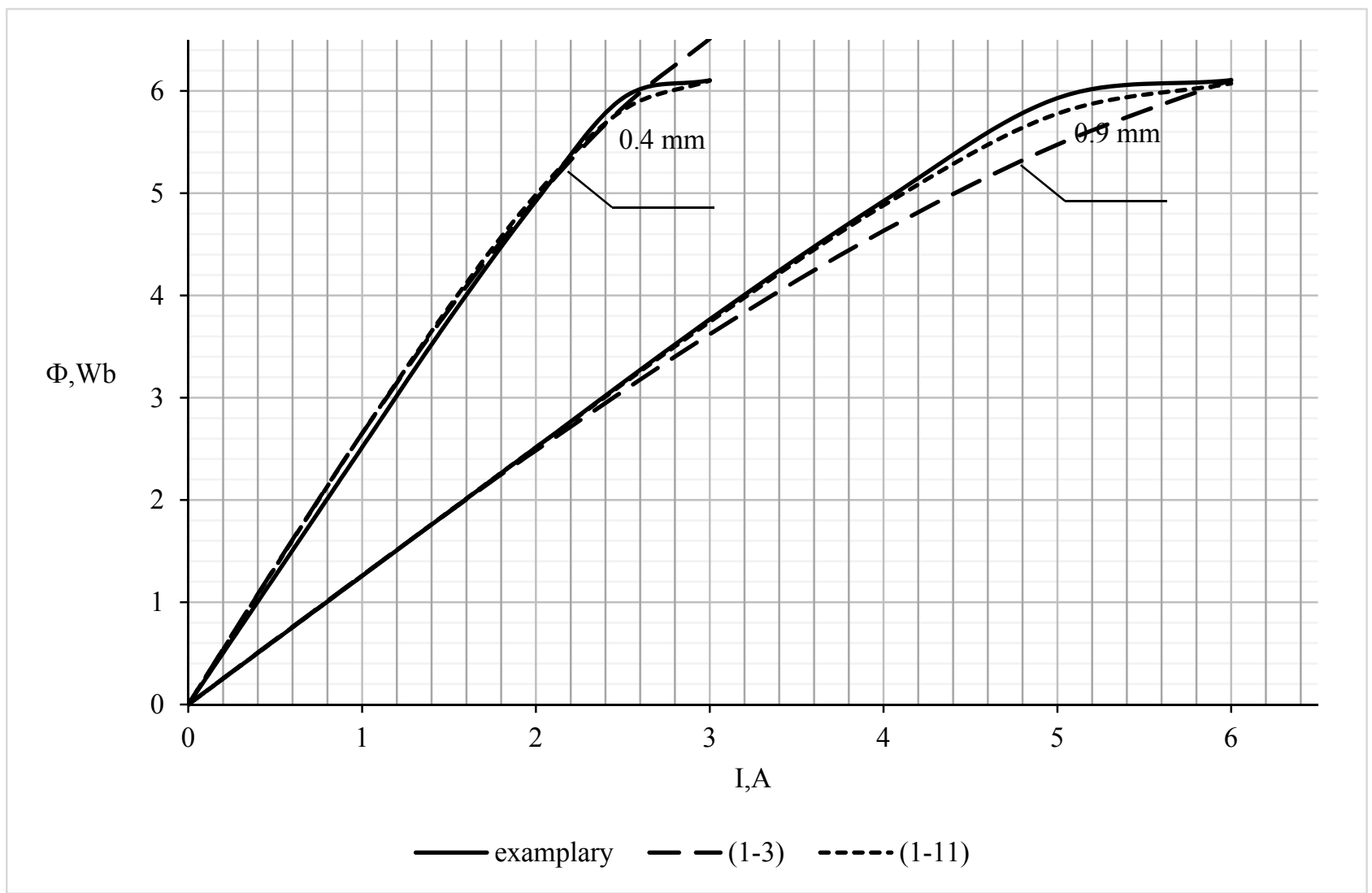

Figure 5. Weber--ampere characteristics of и electric device at preset values of magnetic core gap

\subsection{Full-Scale Experiment}

Now let us demonstrate the principle of determination of weber--ampere characteristic of electric device on the basis of full-scale experiment. The following electric devices were tested: AC electromagnetic realy RPU-1, asynchronous electric motor KD $1-2$, and toroidal transformer STT - 12A.

The specifications of RPU-1 are as follows:

Operating coil has 5000 windings;

Coil-winding wire: $\mathrm{PEV}-2$;

Dimensions (mm): 69×70×37.3;

Weight (kg): 0.25 .

The specifications of asynchronous electric motor KD $1-2$ are as follows:

Supply voltage (V): 127 ;

Operating coil has 3200 windings;

Coil-winding wire: PEV - 2

Power consumption (W): 13;

Dimensions (mm): 4x74x67.5; 
Weight (kg): 0.6.

The specifications of toroidal transformer STT - 12A are as follows:

Outer diameter (mm): 75;

Inner diameter (mm): 40;

Grade of magnetic core steel: E320;

Coil-winding wire: PEV -2 ;

Operating coil has 750 windings;

Weight (kg): 1.1 .

The experiments were performed with the following equipment: laboratory autotransformer Wusley TDGC 8A, two Fluke 289 RMS multimeters, Tektronix 2024b digital oscillograph, PC with the following characteristics:

Wusley TDGC autotransformer:

Power (kW): 2;

Input voltage (V): 220;

Output voltage (V): 0 - 260;

Fluke 289 RMS multimeter:

Basic error (\%): 0.025;

Voltage measurement range: $1 \mu \mathrm{V}-1000 \mathrm{~V}$;

Current measurement range: $10 \mathrm{nA}-10 \mathrm{~A}$;

Resistance measurement range: $0.01 \Omega-500 \mathrm{M} \Omega$;

Memory: 10000 counts;

USB interface;

Tektronix 2024b digital oscillograph:

Transmission range (MHz): 200;

Memory per channel: 2500 samples;

Maximum discretization frequency: $2 \mathrm{GHz}$;

Maximum input voltage: $300 \mathrm{~V}$;

Input impedance: $1 \mathrm{M} \Omega$;

Number of channels: 4 ;

Fast Fourier transform;

USB interface;

Memory: 4 oscillograms.

Flowchart of the assembly is illustrated in Figure 6.

Sinusoidal voltage of preset amplitude is supplied from the output of laboratory autotransformer $L$ to electric device, which is measured by means of multimeter $M$. Shunt $R_{0}$ is connected in series with the coil of electric device. Current shape and harmonics are measured by means of digital oscillograph $O$. The data acquired by the oscillograph and multimeter are transferred to personal computer $P C$, in order to compute weber--ampere characteristic of electric device. 


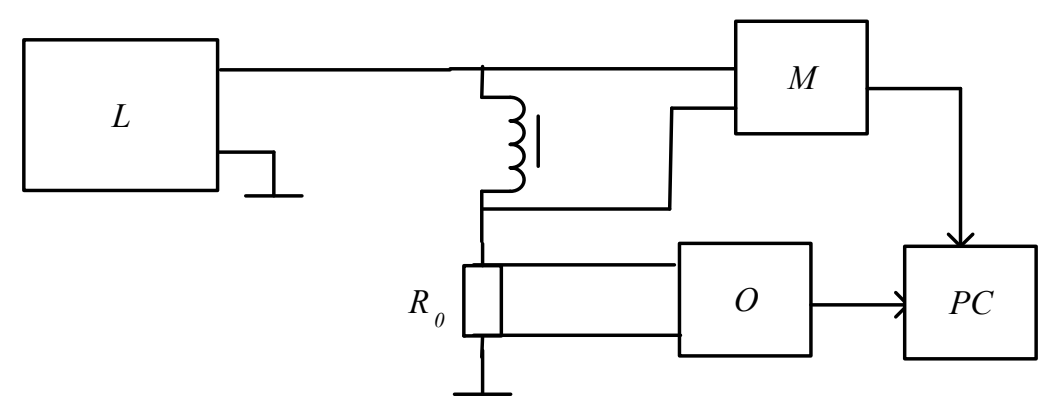

Figure 6. Flowchart of the experimental assembly

Reference weber--ampere characteristics of electric devices were measured by the ammeter--voltmeter method (Komarov, Pokrovskii, Sergeev, and Shikhin, 1984). Measuring coils are applied onto the magnetic cores of electric devices. A coil of electric device is connected to sinusoidal voltage source: laboratory autotransformer. Voltage at the measuring coil and current in the circuit of coil and electric device are measured by multimeters. The obtained results are applied for plotting of weber--ampere characteristic of electric device.

The obtained weber--ampere characteristics of electric devices $\Phi_{\mathrm{n}}\left(I_{\mathrm{n}}\right)$, normalized by the values of maximum voltage on the measuring coil and maximum current in the coil of electric devices are illustrated in Figure 7.

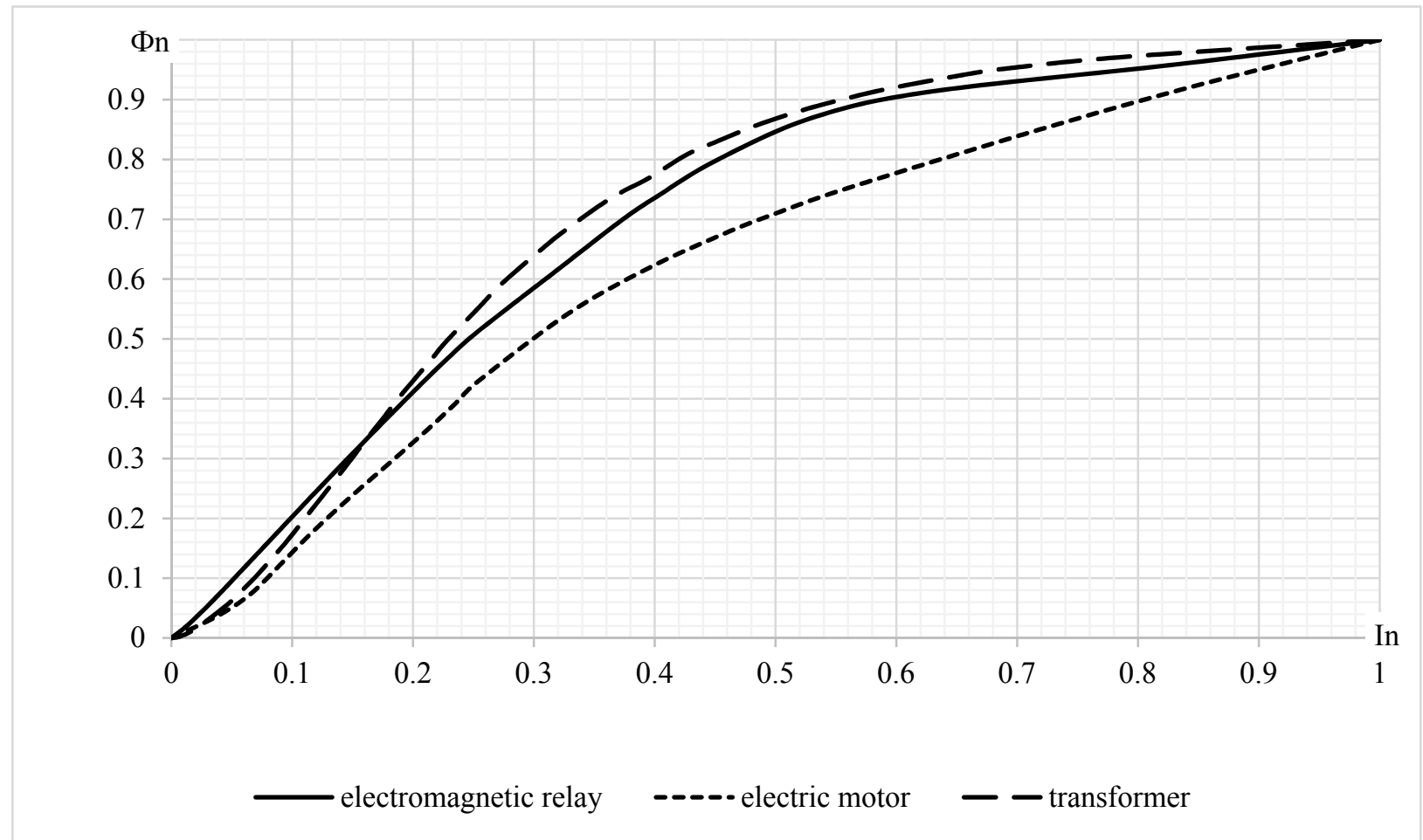

Figure 7. Reference normalized weber--ampere characteristics of electric devices

Calculated weber--ampere characteristics of electric devices are determined by the proposed method based on the solution of inverse problem of harmonic balance. With this aim the amplitude and frequency of the applied voltage, active resistance of coils of electric devices, and the amplitudes of current harmonics in the form of spectrograms are measured.

The results are summarized in Table 1. 
Table 1. Results of preliminary measurements

\begin{tabular}{lccccccc}
\hline Parameter & $U_{a}, \mathrm{~B}$ & $R, \mathrm{OM}$ & $I_{1}, \mathrm{~A}$ & $I_{3}, \mathrm{~A}$ & $I_{5}, \mathrm{~A}$ & $I_{7}, \mathrm{~A}$ & $I_{9}, \mathrm{~A}$ \\
\hline Electromagnetic relay & 260 & 488 & 0.49 & 0.012 & 0.00049 & 0.00042 & 0.00005 \\
Electric motor & 97 & 64.5 & 1.47 & 0.092 & 0.0018 & 0.0014 & 0.00015 \\
Transformer & 200 & 512 & 0.38 & 0.004 & 0.00038 & 0.00004 & 0.000039 \\
\hline
\end{tabular}

Figure 8 illustrates the shapes of current in coils of electric devices.

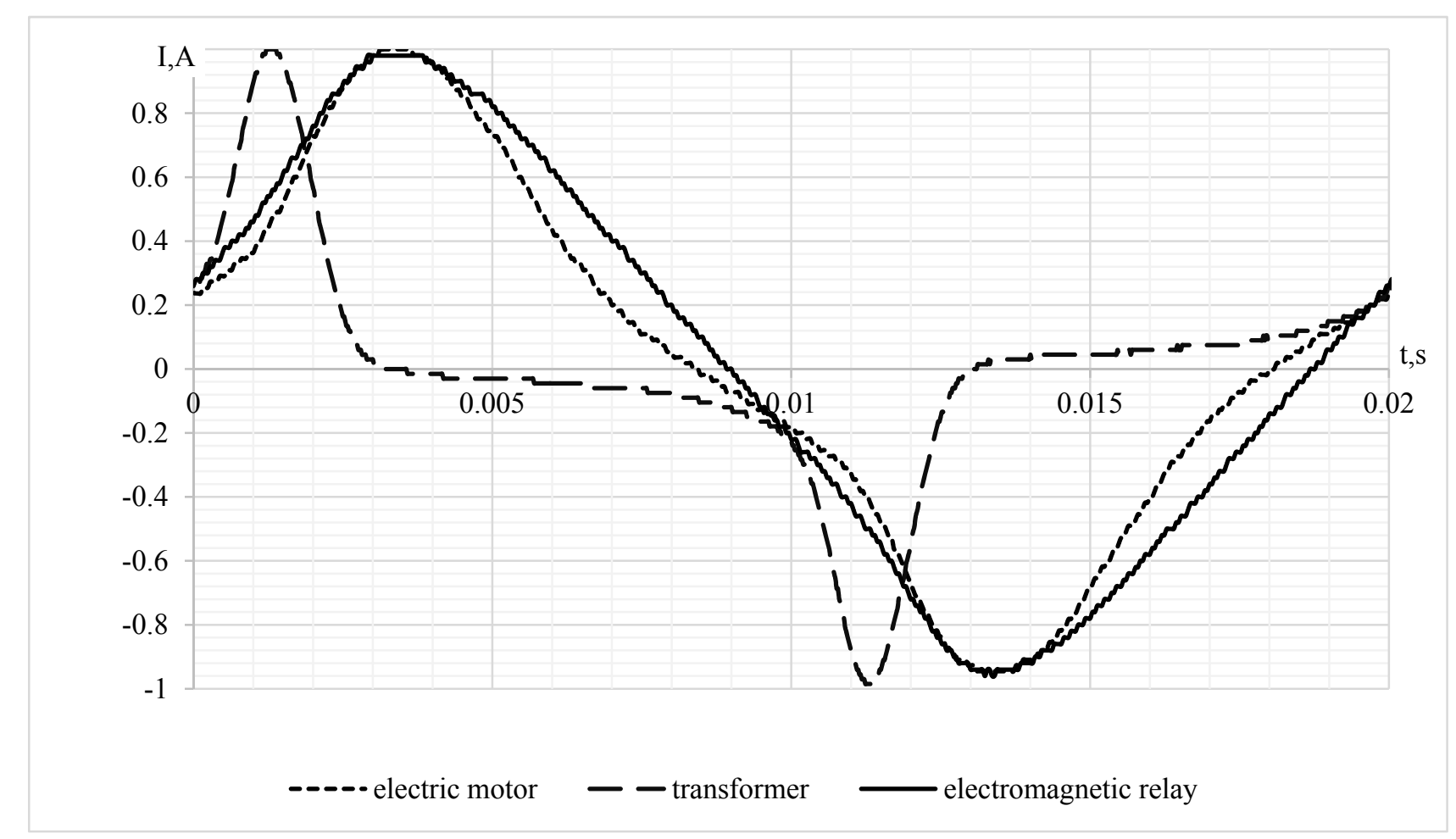

Figure 8. Shapes of current within testing of electric devices

In Figure 8 it can be seen that the current flowing through the coils of electric devices is of various degree of unsinusoidality. The shape of current of toroidal transformer has the highest distortion.

Figures 9--11 illustrate spectrograms of currents in coils of electric devices. The calculations are based on the current harmonics with attenuation not higher than $40 \mathrm{~dB}$. 


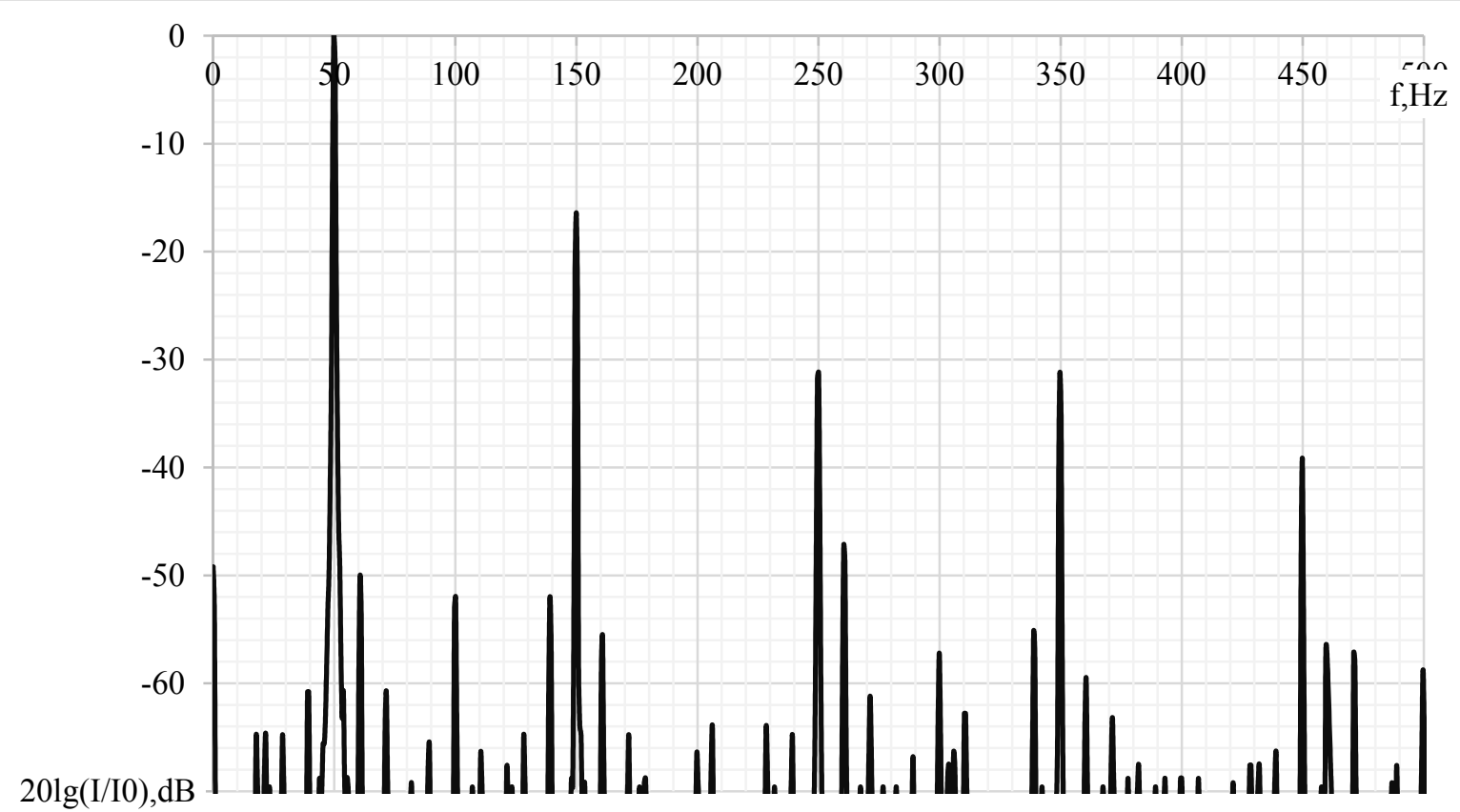

Figure 9. Current spectrum within testing of electromagnetic relay

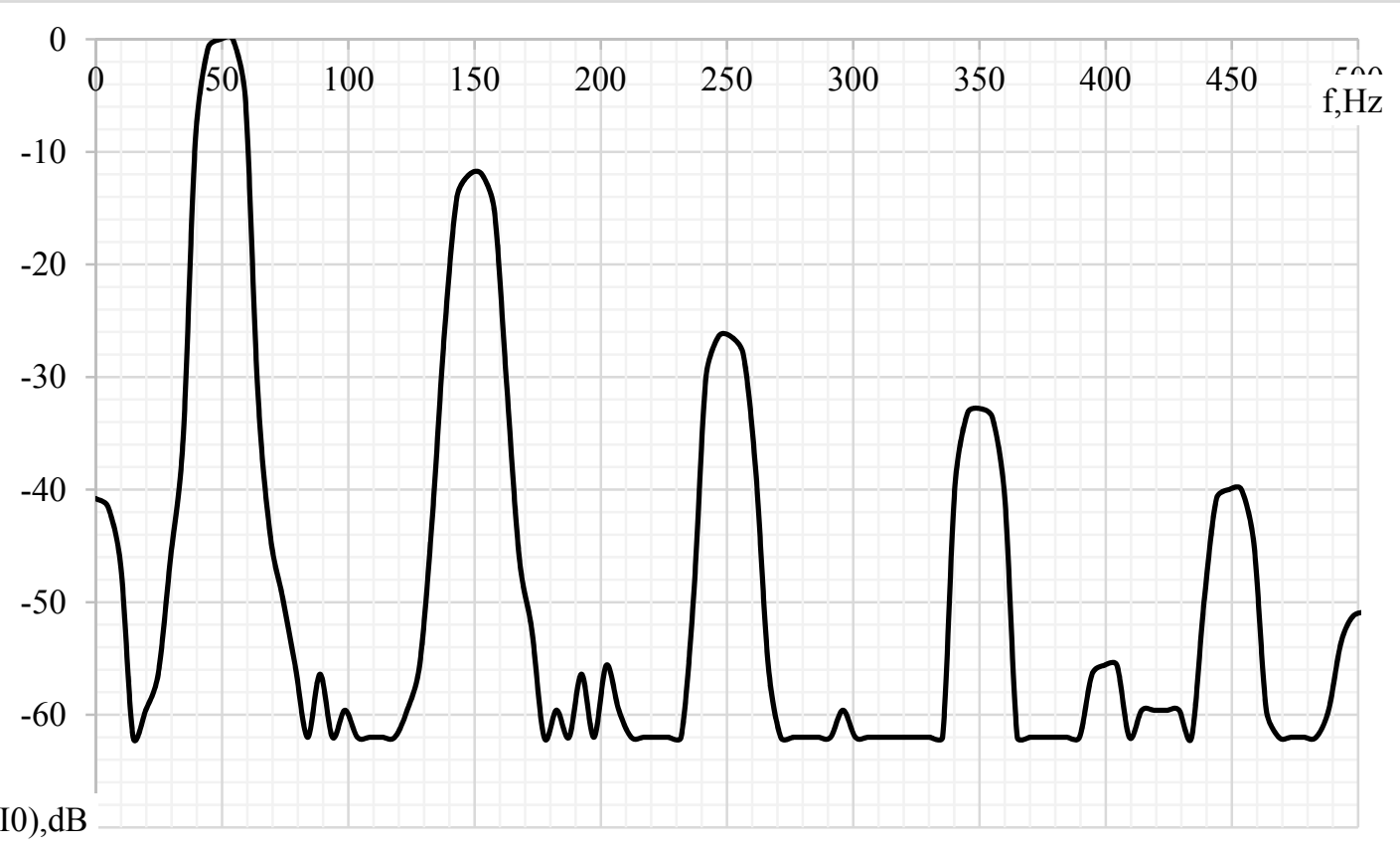

Figure 10. Current spectrum within testing of electric motor 


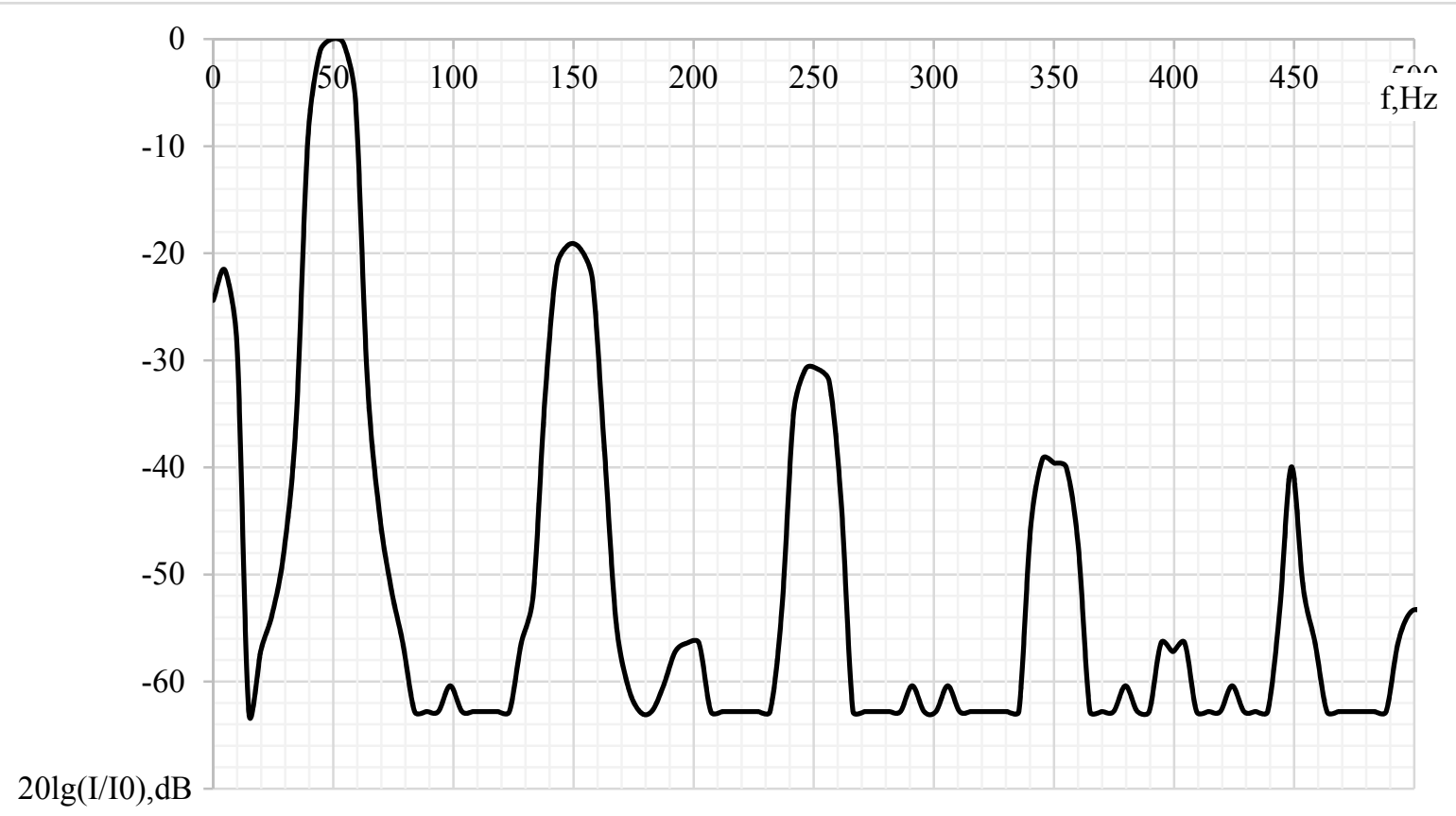

Figure 11. Current spectrum within testing of transformer

The obtained results with the use of the first and the third and from the first to the ninth current harmonics are illustrated in Figs. 12--16.

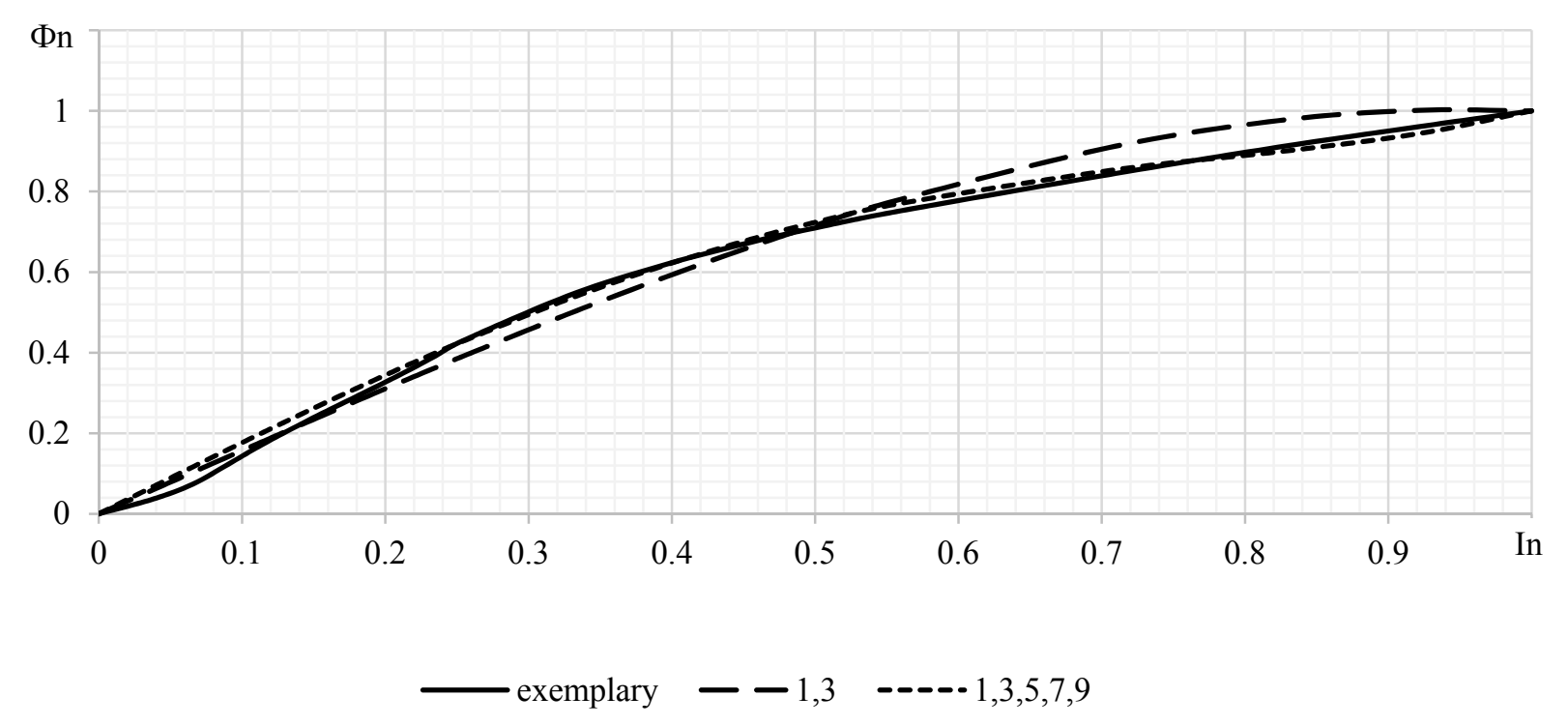

Figure 12. Weber--ampere characteristic of electric motor 


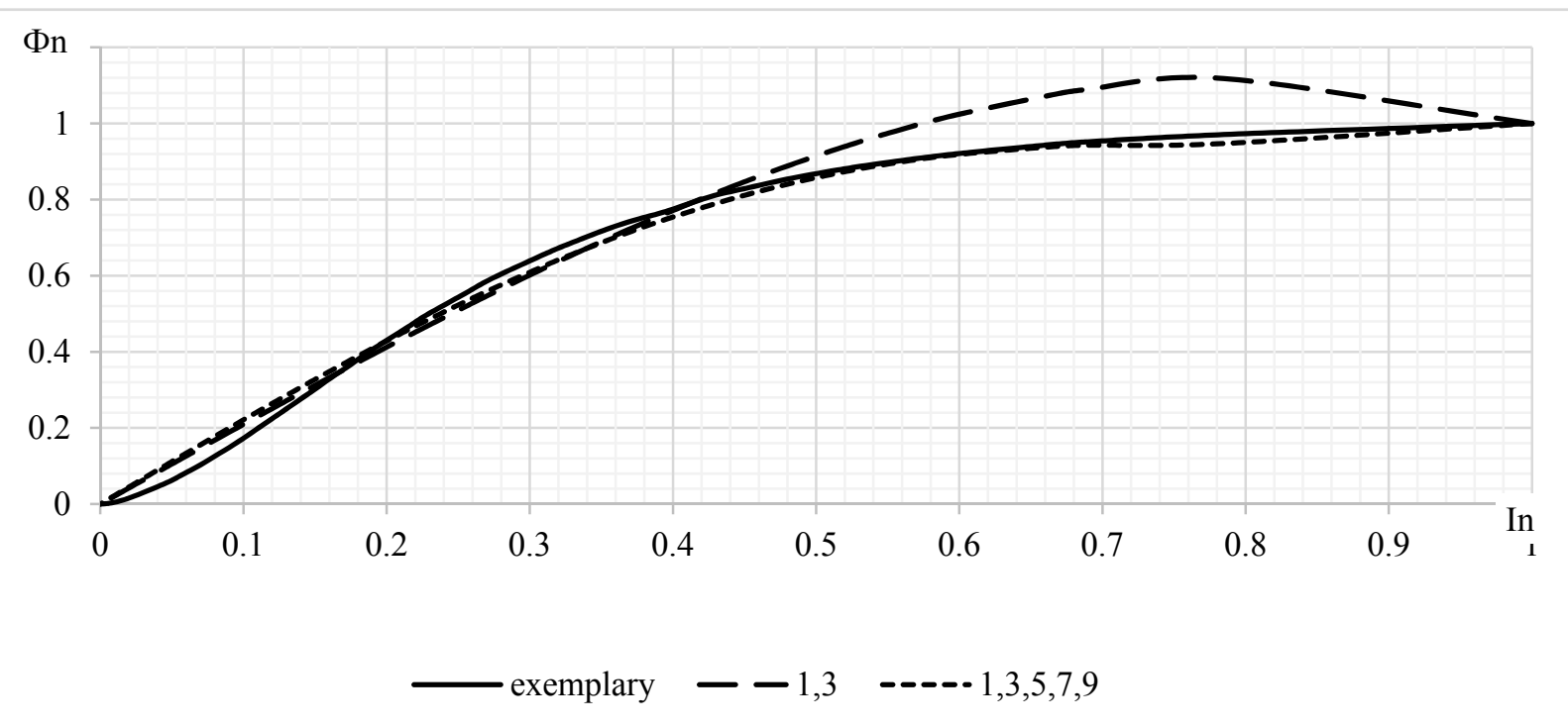

Figure 13. Weber--ampere characteristic of toroidal transformer

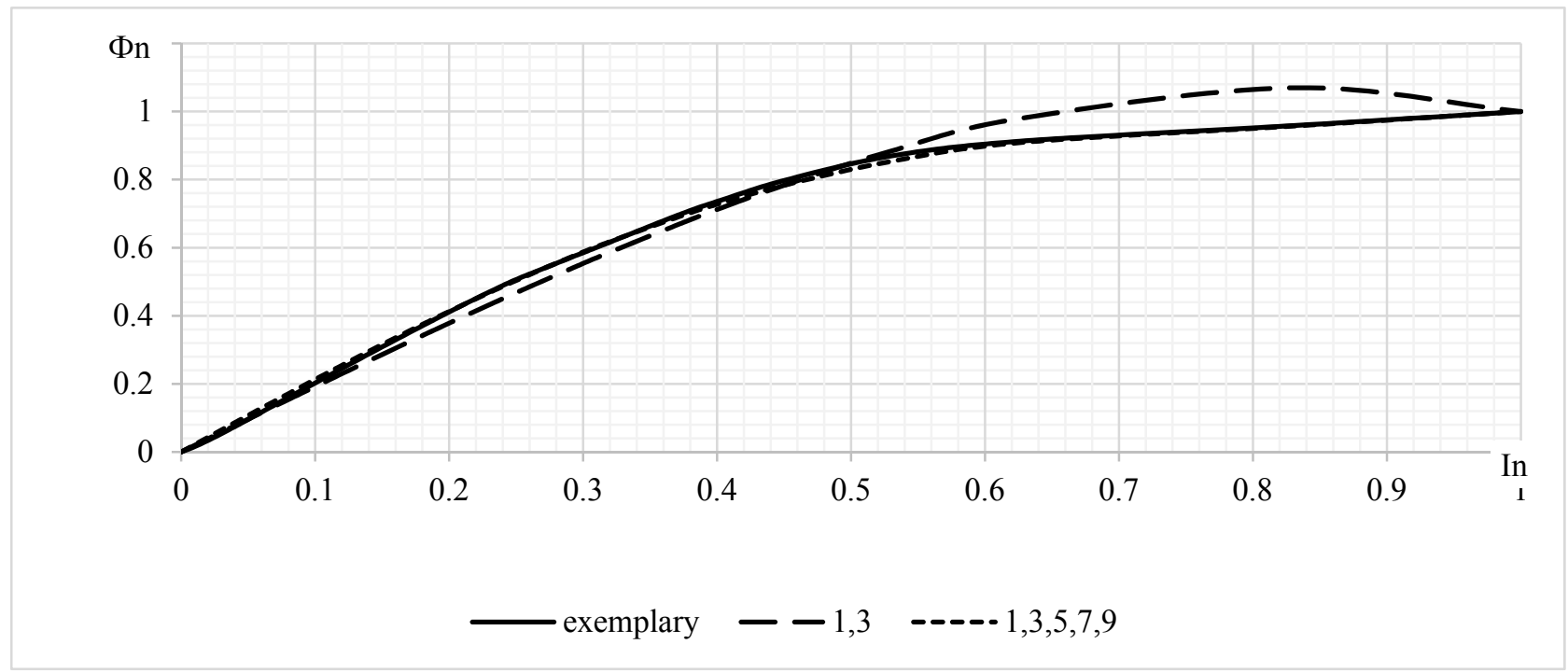

Figure 14. Weber--ampere characteristic of electromagnetic relay

In order to analyze the obtained results it is necessary to calculate the error of determination of weber--ampere characteristics of electric devices. The method of detection of this error (Antonov, Petrov, and Shchelkin,1986; Golt, 2008) is illustrated in Figure 15. 


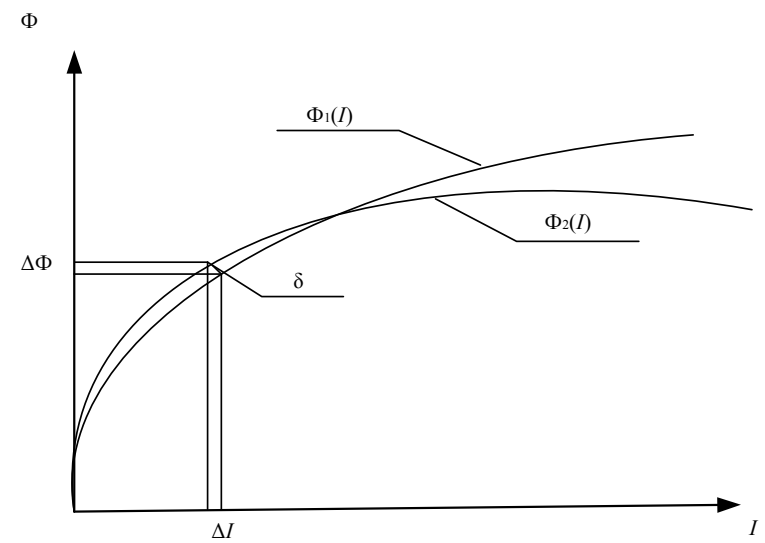

Figure 15. Method of detection of measurement error of weber--ampere characteristic of electric device

A set of points for calculation of the error is determined in reference weber--ampere characteristic $\Phi_{1}(I)$. Then perpendicular is plotted from the reference weber--ampere characteristic $\Phi_{1}(I)$ to the obtained weber--ampere characteristic $\Phi_{2}(I)$, absolute errors for magnetic flow $\Phi$ and current $I$ are determined as projections to appropriate axes. Estimated relative errors of determination of weber--ampere characteristic $\Phi(I)$ for current $I$ and flow $\Phi$, as well as total error are determined as follows:

$$
\delta_{I}=\frac{\Delta I}{I}, \quad \delta_{\Phi}=\frac{\Delta \Phi}{\Phi}, \quad \delta=\sqrt{\delta_{I}^{2}+\delta_{\Phi}^{2}}
$$

The results of calculation of measurement error of weber--ampere characteristic of electric device with different non-magnetic gaps: $0.4 \mathrm{~mm}$ (weber-- ampere characteristic 1 ) and $0.9 \mathrm{~mm}$ (weber-- ampere characteristic 2), obtained by various number of current harmonics are summarized in Table 2 .

Table 2. Errors of determination of weber--ampere characteristics of electric devices with non-magnetic gap

\begin{tabular}{|c|c|c|c|c|c|c|c|c|}
\hline \multicolumn{3}{|c|}{ Maximum considered current harmonic } & 3 & 5 & 7 & 9 & 11 & 13 \\
\hline \multirow{6}{*}{$8^{\circ}$} & weber--ampere & $\delta_{I}$ & 5.3 & 2.8 & 1.3 & 1.4 & 1.1 & 0.9 \\
\hline & characteristic, gap 0.4 & $\delta_{\Phi}$ & 4.5 & 2.4 & 2.8 & 2.4 & 2.1 & 1.8 \\
\hline & $\mathrm{mm}$ & $\delta$ & 7.0 & 3.7 & 3.1 & 2.8 & 2.4 & 2.0 \\
\hline & wher--amnere & $\delta_{I}$ & 6.2 & 5.9 & 5.6 & 4.8 & 4.3 & 4.1 \\
\hline & Weber-ampere & $\delta_{\Phi}$ & 2.1 & 1.3 & 0.9 & 0.9 & 0.8 & 0.8 \\
\hline & & $\delta$ & 6.5 & 6.0 & 5.7 & 4.9 & 4.4 & 4.2 \\
\hline
\end{tabular}

Table 2 summarizes the results of measurement error of weber--ampere characteristics of electric devices in full-scale experiment.

Table 3. Errors of determination of weber--ampere characteristics of electric devices

\begin{tabular}{|c|c|c|c|c|c|}
\hline \multicolumn{3}{|c|}{$\begin{array}{l}\text { Maximum considered } \\
\text { current harmonic }\end{array}$} & Electric motor & Transformer & Electromagnetic relay \\
\hline \multirow{6}{*}{$\begin{array}{l}\partial^{\circ} \\
\stackrel{0}{0}\end{array}$} & & $\delta_{I}$ & 21 & 9 & 18 \\
\hline & 3 & $\delta_{\Phi}$ & 14 & 7 & 12 \\
\hline & & $\delta$ & 25 & 11 & 22 \\
\hline & & $\delta_{I}$ & 2.5 & 2.1 & 1.2 \\
\hline & 9 & $\delta_{\Phi}$ & 1.8 & 1.5 & 0.9 \\
\hline & & $\delta$ & 3.1 & 2.6 & 1.5 \\
\hline
\end{tabular}

In Table 3 it can be seen that the use of the first and the third current harmonics provides deviation from 
reference characteristic in overall interval of the weber--ampere characteristics, and the use of the current harmonics from the first to the ninth makes it possible to achieve acceptable accuracy of det3ermination of weber--ampere characteristics of electric devices.

The obtained results demonstrate that the successful application of the method considerably depends on correct selection of the number of harmonics. In order to determine the required number of current harmonics involved in the calculation of coefficients $k_{m}$ of approximating equation of weber--ampere characteristic (9) we apply the known approach (Lvovsky, 1988; Mason, Mukhopadhyay \& Jayasundera, 2013; Freund, Mohr, Wilson, 2010).

Successively increasing the number of current harmonics we control the variation of residual dispersion:

$$
S_{\mathrm{res}}^{2}=\frac{\sum_{i=1}^{n}\left(\Phi_{i}-\overline{\Phi_{i}}\right)^{2}}{n-q-1},
$$

where $n$ is the number of points, where the compared reference values of flow $\overline{\Phi_{i}}$ and calculated $\Phi_{i}$ were measured, $q$ is the number of the used current harmonics.

Termination of addition of current harmonics was determined by verification of hypothesis about the absence of difference between residual dispersions $S_{\text {res }}^{2}$. With this aim the Fischer criterion was applied, according to which the calculated Fischer criterion is determined as follows:

$$
F_{\mathrm{p}}=\frac{S_{\text {res( } \mathrm{j})}^{2}}{S_{\text {res }(\mathrm{j}+1)}^{2}}
$$

and then is compared with the critical value of Fischer criterion for the following number of degrees of freedom $f_{1}=n-1 ; f_{2}=n-q-1$.

When the condition $F_{\mathrm{p}} \leq F_{\mathrm{кр}}$ is satisfied, the addition of current harmonics terminates.

The results of determination of the required number of current harmonics at confidence level $\alpha=5 \%$ are summarized in Table 4.

Table 4. Determination of number of current harmonics

\begin{tabular}{cccccccc}
\hline Parameter & $2 \mathrm{p}+1$ & $S_{\text {ост }}^{2}$ & $p$ & $f_{1}$ & $f_{2}$ & $F_{\mathrm{p}}$ & $F_{\text {кр }}$ \\
\hline & $\mathbf{3}$ & 0.091 & 2 & 18 & 16 & - & - \\
weber - ampere & $\mathbf{5}$ & 0.022 & 3 & 18 & 15 & 4.09 & 2.39 \\
characteristic, gap & $\mathbf{7}$ & 0.0081 & 4 & 18 & 14 & 2.75 & 2.44 \\
0.4 mm & $\mathbf{9}$ & 0.0031 & 5 & 18 & 13 & 2.67 & 2.51 \\
& $\mathbf{1 1}$ & 0.0012 & 6 & 18 & 12 & 3.00 & 2.6 \\
weber - ampere & $\mathbf{3}$ & 0.00072 & 7 & 18 & 11 & 1.43 & 2.71 \\
characteristic, gap & $\mathbf{5}$ & 0.063 & 2 & 18 & 16 & - & - \\
0.9 mm & $\mathbf{7}$ & 0.021 & 3 & 18 & 15 & 3.00 & 2.39 \\
& 9 & 0.0084 & 4 & 18 & 14 & 2.50 & 2.44 \\
\hline
\end{tabular}

From Table 4 it follows that for the first weber--ampere characteristic it is required to apply from the first to the eleventh odd current harmonics, and for the second weber--ampere characteristic from the first to the seventh harmonics.

\section{Conclusions}

The work analyzes known methods of measurements of weber--ampere characteristic of electric devices, their unsuitability for determination of weber--ampere characteristic of operating cycle is revealed. In order to obtain the set purpose it is proposed to apply solution of inverse problem of harmonic balance. Mathematical analysis of solution of inverse problem of harmonic analysis has been performed, simulation and full-scale experiments have been carried out using mathematical model of the Micro-Cap simulator and three types of electric devices 
(electromagnetic relay, electric motor, and toroidal-core transformer). The experimental results make it possible to state that the proposed method of measurement of weber--ampere characteristic based on the solution of inverse problem of harmonic balance facilitates obtaining of characteristic of electric device with the error not exceeding $3 \%$. This method can be applied for testing of electric devices both within their fabrication and operation.

\section{Acknowledgments}

The work was supported by the Project No. 1.2690.2014/K titled Methods of solution of inverse problems of diagniostics of complex systems (in engineering and medicien) based on full-scal and simulation experiments, performed in the scope of designing portion of Federal Program on the premises of Center of Collective Usage "Diagnostics and poer saving electric equipment", Platov South-Russian State Polytechnic University (NPI).

\section{References}

Amelina, M. A., \& Amelin, S. A. (2007). Micro-Cap 8 Simulator. Moscow, Goryachaya Liniya, Telekom.

Antonov, V. G., Petrov, L. M., \& Shchelkin, A. P. (1986). Measurement tools of magnetic parameters of materials. Leningrad: Energoatomizdat.

Bessonov, L. A. (1978). Theoretical foundations of electric engineering: Electric circuits. Textbook for students of electric engineering and instrument building specialties of higher schools. Moscow, Vysshaya Shkola.

Direct-current Magnetic Measurements for Soft Magnetic Materials. ASTM International, 1970.

Frank, W. (2012). Roller Electric and Magnetic Measurements and Measuring Instruments. HardPress.

Georgi, P. (2012). Tolstov Fourier Series. Courier Dover Publications. http://dx.doi.org/ 10.1115/1.3630087

Igor, K., \& Valerii, Z. (2010). Radoslaw Kasperek Electrotechnical Systems. CRC Press. http://dx.doi.org/ $10.1201 / 9781420087109$

Joachim Vester Simulation elektronischer Schaltungen mit MICRO-CAP. Springer-Verlag, 2009. http://dx.doi.org/ 10.1007/978-3-8348-9333-8

Kiefer, I. I. (1969). Testing of ferromagnetic materials. Moscow, Energia, 272-273.

Komarov, E. V., Pokrovskii, A. D., Sergeev, V. G., \& Shikhin, A. Ya. (1984). Testing of magnetic materials and systems. Moscow, Energoatomizdat.

Lvovsky, E. N. (1988). Statistic methods of development of empiric equations. textbook for higher schools. Moscow: Vysshaya Shkola,

Mason, A. S. C., Mukhopadhyay, K. P., \& Jayasundera, N. B. (2013). Sensing Technology: Current Status and Future Trends. - Springer Science \& Business Media. http://dx.doi.org/ 10.1007/978-3-319-02318-2

Michael, C. (2008). Golt Magnetic and Dielectric Properties of Magneto-dielectric Materials. ProQuest. 150 c.

Richard, H. (2010). Enns It's a Nonlinear World. Springer Science \& Business Media. http://dx.doi.org/ 10.1007/978-0-387-75340-9

Richard, H. Enns, \& George, C. (2000). McGuire Nonlinear Physics with Maple for Scientists and Engineers. Springer Science \& Business Media. http://dx.doi.org/10.1007/978-1-4612-1322-2

Robert, J. (1994). Lopez Maple via Calculus. Springer Science \& Business Media. http://dx.doi.org/10.1007/978-1-4612-0267-7

Rudolf, J., Freund, D. M., \& William, J. W. (2010). Statistical Methods - Academic Press.

Sakhavova, A. A., Shirokov, K. M., \& Yanvarev, S. G. (2013). Application of indirect determination of weber-ampere characteristics in automated system of sensorless diagnostics of electromagnetic mechanisms. Modern problems of science and education. Retrieved from http://www.science-education.ru/111-10234

Singh, S. K. (2003). Industrial Instrumentation \& Control. Tata McGraw-Hill Education.

Song, X., Suting, C., Zhanming, W., \& Jingming, X. (2013). Unifying Electrical Engineering and Electronics Engineering. Springer Science \& Business Media. http://dx.doi.org/ 10.1007/978-1-4614-4981-2

\section{Copyrights}

Copyright for this article is retained by the author(s), with first publication rights granted to the journal.

This is an open-access article distributed under the terms and conditions of the Creative Commons Attribution license (http://creativecommons.org/licenses/by/3.0/). 